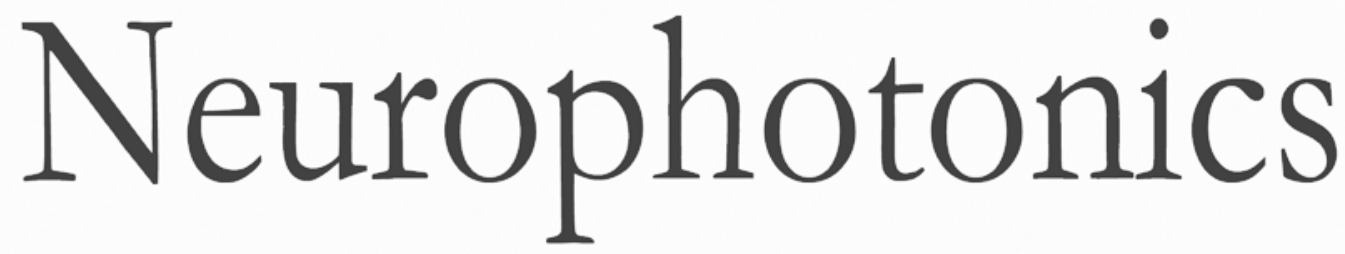

\title{
Isolating the effects of surface vasculature in infant neuroimaging using short-distance optical channels: a combination of local and global effects
}

Lauren L. Emberson

Stephen L. Crosswhite

James R. Goodwin

Andrew J. Berger

Richard N. Aslin 


\title{
Isolating the effects of surface vasculature in infant neuroimaging using short-distance optical channels: a combination of local and global effects
}

\author{
Lauren L. Emberson, ${ }^{a, b, c, \star}$ Stephen L. Crosswhite, ${ }^{a}$ James R. Goodwin, ${ }^{d}$ Andrew J. Berger, ${ }^{d}$ and \\ Richard N. Aslin ${ }^{a, b}$ \\ aUniversity of Rochester, Brain and Cognitive Sciences, Meliora Hall, Box 270268, Rochester, New York 14627, United States \\ bUniversity of Rochester, Rochester Center for Brain Imaging, 430 Elmwood Avenue, Box 278917, Rochester, New York 14627, United States \\ 'Princeton University, Peretsman-Scully Hall, Psychology Department, Princeton, New Jersey 08544, United States \\ 'University of Rochester, The Institute of Optics, Wilmot Building, 275 Hutchinson Road, Rochester, New York 14627, United States
}

\begin{abstract}
Functional near-infrared spectroscopy (fNIRS) records hemodynamic changes in the cortex arising from neurovascular coupling. However, (noninvasive) fNIRS recordings also record surface vascular signals arising from noncortical sources (e.g., in the skull, skin, dura, and other tissues located between the sensors and the brain). A current and important focus in the fNIRS community is determining how to remove these noncortical vascular signals to reduce noise and to prevent researchers from erroneously attributing responses to cortical sources. The current study is the first to test a popular method for removing signals from the surface vasculature (removing short, $1 \mathrm{~cm}$, channel recordings from long, $3 \mathrm{~cm}$, channel recordings) in human infants, a population frequently studied using fNIRS. We find evidence that this method does remove surface vasculature signals and indicates the presence of both local and global surface vasculature signals. However, we do not find that the removal of this information changes the statistical inferences drawn from the data. This latter result not only questions the importance of removing surface vasculature responses for empiricists employing this method, but also calls for future research using other tasks (e.g., ones with a weaker initial result) with this population and possibly additional methods for removing signals arising from the surface vasculature in infants. @ 2016 Society of Photo-Optical Instrumentation Engineers (SPIE) [DOI: 10.1117/1.NPh.3.3.031406]
\end{abstract}

Keywords: functional near-infrared spectroscopy; infant; neurovascular; surface vasculature; short channel.

Paper 15055SSR received Dec. 12, 2015; accepted for publication Mar. 8, 2016; published online Apr. 19, 2016.

\section{Introduction}

Since the advent of noninvasive functional neuroimaging of the human brain in the mid-1990s, the application of functional magnetic resonance imaging (fMRI) to studies of young infants has been a goal of developmental cognitive neuroscientists. That goal has largely remained unfulfilled for a variety of technical reasons, most notably the requirement of rigid head stabilization. Although a handful of fMRI studies have been conducted with infants (see Ref. 1 for a systematic review of this literature), they have involved a sleeping preparation that is successful in reducing motion artifacts but prevents studies of sensory stimulation in the visual modality. Since the mid-2000s, the application of functional near-infrared spectroscopy (fNIRS) has been championed as a viable alternative to fMRI for studies of awakebehaving infants. ${ }^{2-4}$ Because the optical fibers that deliver NIR light to the scalp and record the modulations in NIR light returning from the underlying cortex are attached to the infant's head with a tight-fitting cap, there are minimal motion artifacts. Thus, over the past decade, fNIRS has become an essential alternative to fMRI and has kick-started the investigation of functional neural development starting in infancy (see reviews by Refs. 1 and 5-8).

Despite the promise of fNIRS, a major technical limitation of the method is that the optical signal must pass through noncortical tissues in order to record hemodynamic changes in the

*Address all correspondence to: Lauren L. Emberson, E-mail: lauren. emberson@princeton.edu brain. As a result, the optical signal travels from the emitter located on the scalp through the layers of skin, skull, dura, cerebral spinal fluid, and so on before reaching the target cortical tissue and then returns through these layers back to the detector. This double-pass route for NIR photons picks up noncortical signals that contaminate the optical signals from the targeted region of cortex, thereby rendering fNIRS extremely sensitive to contamination from vascular changes arising from noncortical sources.

We will refer to vascular changes not arising from cortical neurovascular coupling as surface vasculature signals. The term neurovascular coupling specifically refers to localized circulatory responses in the cortex in direct response to neural activity in that same region of the cortex. It is important to note that noncortical circulatory signals can also be triggered by neural activity (e.g., blushing, sympathetic nervous system responses to a perceived threat). Thus, while circulatory responses in the surface vasculature surrounding the brain are often considered basic physiological responses, like blood pressure, signals in the surface vasculature can originate from cortical activity through an indirect path. However, while this is a type of relationship between the circulatory signal and the brain, these signals are not the type of signals that is being referred to by the term neurovascular coupling; nor are they the target of the field of cognitive neuroscience. Thus, like more physiological signals in the circulatory system, the goal of measuring 
short-channel signals directly is to provide a means to remove any noncortical circulatory signals from the long-channel recordings. Therefore, in this paper, we do not make a distinction between the possible origins of circulatory signals in the surface vasculature. Rather, for the purposes of simplifying the discussion in the current paper, we will simply refer to signals in the surface vasculature as a singular term to refer to any noncortical circulatory signal that could be embedded in signals recorded from long NIRS channels.

The inclusion of surface vasculature signals in fNIRS recordings is important to address as it can give rise to both type I and type II statistical errors (the incorrect rejection of the null hypothesis and a false negative or the failure to reject an incorrect null hypothesis, respectively). At best, the contamination of fNIRS signals by the surface vasculature will simply contribute noise that may obscure the signal arising from neurovascular coupling. For example, spontaneous Mayer waves $(0.1 \mathrm{~Hz})$, changes in blood pressure $(0.025$ to $0.35 \mathrm{~Hz})$, and changes in blood oxygenation with respiration $(0.1$ to $0.5 \mathrm{~Hz})$ can add noise to the signal that may swamp hemodynamic changes arising from neural activity. At worst, signals from the surface vasculature could vary with an experimental task. For example, when comparing experimental conditions that vary in task difficulty or emotional salience, participants may experience changes in blood pressure and heart rate that are task dependent. These task-related fluctuations in vascular activity could be erroneously interpreted as the task involvement of the cortical region sampled by the channel. Thus, surface vascular signals can give rise to both type I and type II errors in fNIRS studies and present a significant hurdle to the widespread adoption of this method. See the excellent work by Gagnon and colleagues for a quantification of the cortical versus noncortical contributions to the typical fNIRS signal. ${ }^{9}$

In response to this problem, researchers have focused on developing a solution that directly measures these noncortical vascular signals and removes them from the signals originating from a targeted cortical area. Saager and Berger ${ }^{10}$ reasoned that if fNIRS channels with the typical emitter-detector separation of $3 \mathrm{~cm}$ (chosen so that returning photons have traveled into the cortex) contained vasculature changes arising from both cortical sources (arising from neurovascular coupling) and noncortical (surface vasculature) sources, then fNIRS channels with a shorter emitter-detector separation of $\sim 1.0 \mathrm{~cm}$ could be used to record only the surface vascular changes. In other words, Saager and Berger ${ }^{10}$ proposed the use of a short-distance optical channel or short channel to directly measure only the surface vasculature signals and then to use this signal to remove the contribution of the surface vasculature from the longer fNIRS channel (long channel, Fig. 1). To be clear, there are a variety of experimental designs that could, in principle, mitigate these contaminating effects of surface vascular signals (especially in the case where suface vasculature contributes noise and leads to a type II error). For example, just as event related potentials (ERP) studies collect many epochs time locked to the onset of a stimulus, and employ signal averaging to cancel out spontaneous background electroencephalography (EEG), one could simply present many epochs of the stimuli in an fNIRS study and employ the same signal averaging logic to these analyses. However, this experimental design will only eliminate surface vasculature signals that are uncorrelated with the experimental task (e.g., arousal induced by an experimental manipulation can modulate the surface vasculature in a task-dependent fashion).

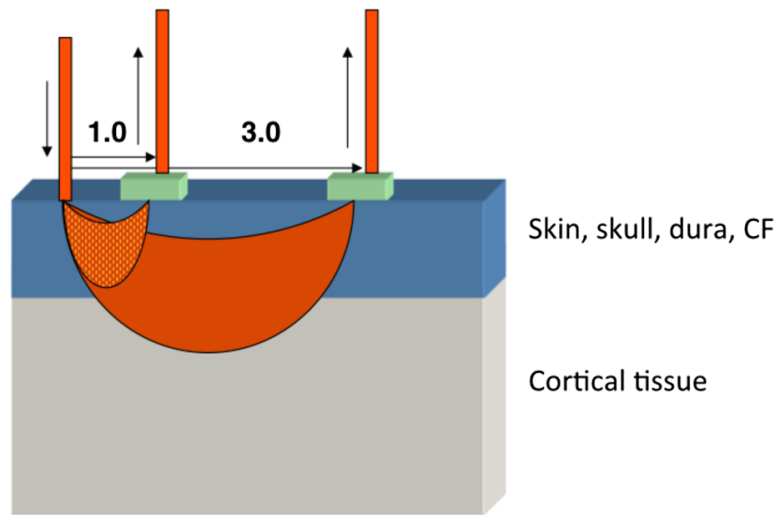

Fig. 1 Depiction of the basic concepts behind short-channel NIRS recordings. While the exact length of short and long channels varies across experiments and research groups, short channels are typically $1 \mathrm{~cm}$ (as depicted here), and long channels are typically 2 to $3 \mathrm{~cm}$ (depicted here at $3 \mathrm{~cm}$ consistent with the current experiment). The banana-shaped paths are approximate volumes explored by detected photons. CSF, cerebral-spinal fluid. Distances are expressed in centimeters $(\mathrm{cm})$.

Moreover, given the slow time course of the hemodynamic response (tens of seconds compared to a few hundred milliseconds for EEG), there are logistical reasons why signal averaging is not as practical for fNIRS as for ERPs (i.e., infants cannot be tested for more than 10 to $20 \mathrm{~min}$ ).

There have been a number of demonstrations of the effectiveness of using short fNIRS channel recordings to remove surface vasculature signals from typical, long fNIRS channels. Saager and Berger ${ }^{10,11}$ showed, via simulations, phantoms, and actual fNIRS recordings from adults, that short-channel signals could be used to remove variation in the regular fNIRS or long channel that sampled from the cortex and specifically that removing short-channel information could improve the signal-to-noise ratio of the long-channel recordings by up to a factor of 3 . Zhang and colleagues ${ }^{12,13}$ found that an adaptive filtering technique, employing signals from the short channels, reduced the contrast-to-noise ratio in both simulations and recordings of visual responses in adults. Gagnon and colleagues ${ }^{14}$ also demonstrated the effectiveness in removing signals from short channels using both simulations and experimental recordings with adults. Moreover, they found that by employing two short channels for each long fNIRS channel (one each for the source and detector) that noise reduction is increased, suggesting the presence of highly local effects in systematic vasculature (see also Ref. 15).

Despite these existence proofs in support of the short-channel technique to remove surface vasculature signals, there have been few demonstrations of the empirical advantage of collecting near-channel fNIRS data. In order words, while demonstrations of reductions in noise in the signal are clearly important, for researchers who employ fNIRS to make inferences about underlying neural activity to be convinced of the importance of the technique, it must be demonstrated that any changes in signal quality also go on to have an effect in statistical tests. Only a single and very recent paper has demonstrated that removing short-channel information affects statistical outcomes in adults. ${ }^{16}$ The current paper also employs standard statistical techniques to compare statistical results before and after the removal of short-channel signals.

In addition to providing one of the first direct tests of whether removing short-channel information changes statistical inference, 
the current paper is the first to investigate the nature of shortchannel recordings in young infants. All previous papers have employed simulations, phantoms, or adult fNIRS recordings; however, a large proportion of fNIRS researchers study developing populations, and it is unknown whether short-channel recordings are similarly beneficial in infants (i.e., whether there are significant signal changes after the removal of the short channel in young infants). Specifically, there are at least three factors that may influence the effectiveness of the short-channel technique in infants. First, there is significant development in skull thickness starting early in life, ${ }^{17}$ which changes the distance between the scalp and the cortex. Second, while still controversial, there appear to be notable developmental changes in the hemodynamic response (for reviews, see Refs. 1 and 18). Third, it is possible with the development of the circulatory system that the characteristics of surface vasculature signals change with age. Combined with a decrease in the amplitude of the circulatory response produced by neurovascular coupling, the relative contributions of surface versus cortical vascular changes to fNIRS recordings may shift with age (see review by Ref. 19).

Thus, the goal of the present study is to analyze fNIRS data from human infants that were obtained from both long and short channels ( 3 and $1 \mathrm{~cm}$, respectively) and to determine whether removing short-channel signals can be used to change statistical inferences made with data from the long channels. We chose $3 \mathrm{~cm}$ for the long channels as this distance is commonly used in both adults and infants for recording cortical activation (see Refs. 6 and 20 for validation studies of $3 \mathrm{~cm}$ channels in infants). The choice of $1 \mathrm{~cm}$ for the short channel is both because many seminal studies in this area have investigated short-channel distances around $1 \mathrm{~cm}^{10,11,12,13}$ as well as constraints on the probe design arising from the diameter of the probe tips (i.e., channels substantially shorter than $1 \mathrm{~cm}$ would have required a redesign of the probe tips). While it is possible that even shorter channel distances could be better suited for infants than adults, we opted to conform to the bulk of the previous literature (i.e., employing $1 \mathrm{~cm}$ as with many previous studies) over the exploration of different shortchannel distances. We complemented this decision with careful checks to make sure that cortical responses are not seen in the short channels (see Sec. 5). We also examine whether spatial proximity is important for short-channel removal (i.e., whether there are local and/or global surface vascular signals in infant fNIRS channels). Importantly, we compare any changes resulting from short-channel removal to a bootstrapped baseline to determine whether signal change is beyond chance. Overall, this study is intended to determine whether any short channel data are advantageous for removing effects of surface vasculature in young infants during a standard experimental paradigm.

\section{Setup of the Experiment and Functional Near-Infrared Spectroscopy Recordings}

\subsection{Functional Near-Infrared Spectroscopy Recordings}

fNIRS recordings were obtained using a CW6 from TechEn Instruments (Milford, Massachusetts). Four fiber optic cables delivered NIR light to the scalp of the infant (emitters, 690 and $830 \mathrm{~nm}$ ) and eight fiber optic cables detected the reflected light on the scalp. These fibers were situated against the infant's scalp using a custom-made headband that secured fibers to the scalp of infants in as comfortable a fashion as possible and was resistant to motion artifacts (i.e., moving against the scalp when the infants moved). Crucially, the detectors were placed at two different distances from the emitters: long channels were formed by placing detectors $3 \mathrm{~cm}$ from the emitters, and short channels were formed by placing detectors at $1 \mathrm{~cm}$ from the emitters. The relative length of the long and short channels was designed to record the neurovascular response on the surface of the infant cortex $^{6}$ and to record vascular changes in the area of the head between the scalp and the surface of the cortex, respectively. The probe configuration was designed to have symmetric long channels that provide concentrated coverage from a single cortical region. Then, each emitter in this configuration is paired with a single short channel so that local surface vasculature signals can be removed from the long channels arising from that emitter; see Fig. 2 for a schematic of the channel configuration along with channel numbers. The probe design also balances the constraints of having as complete coverage as possible for a single cortical region, a short channel for each emitter, and a limited number of probes that can be accommodated by infants and are allowable by the CW6 from TechEn. Overall, this configuration is ideal for identifying and removing local surface vascular changes that may be contaminating signals from the cortex by including a short channel for each emitter in a symmetric long-channel probe design.

\subsection{Recruited Sample}

Twelve infants were recruited for the current experiment: mean age $=6.1$ months, $\mathrm{SD}=0.58$ months, range $=5.2$ to 6.9 months. The sample had 5 males and infants were identified as 11 being Caucasian with the 12th infant identified as Other, 11 as being non-Hispanic with the 12th infant left unreported. Infants were recruited through the Rochester Baby Lab and had no known visual and hearing difficulties, no major surgeries, or health problems, and were born at 36 weeks gestation or later. All caregivers provided consent for participation in accordance with the institutional review board of the University of Rochester.

\subsection{Experimental Procedure}

After consent, families were taken into an experimental room where the caregiver and infant were seated on a chair in front of a 17-in. video display monitor and separated from the rest of the room with floor-to-ceiling length black curtains. The purpose of the curtains is to reduce visual and auditory

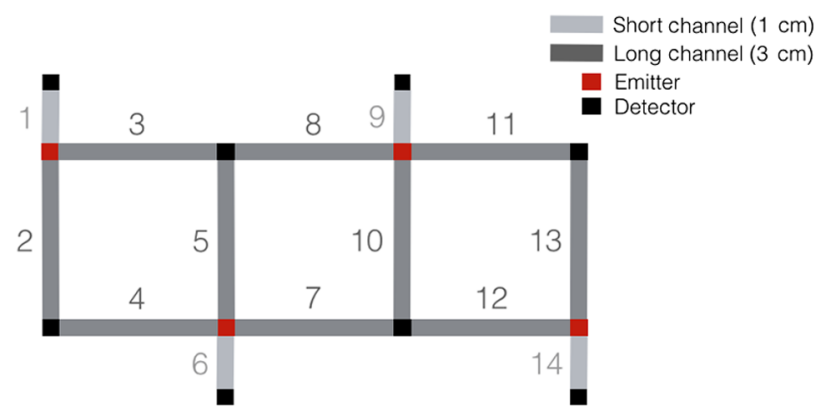

Fig. 2 Channel configuration with long and short channels created from the placement of detectors that have different distances (3 and $1 \mathrm{~cm}$, respectively) from the emitters. There is one short channel for each emitter and one to two long channels that allow for the detection and removal of surface vascular changes from the long channels, which will additionally contain neurovascular changes. Channel numbers are labeled. 
distraction for the infant and encourage them to look at the monitor. Infants were outfitted with the custom probe holder. The holder was placed such that the probes were located starting at the inion and moving more dorsally (see Fig. 3). This position was determined to maximize the likelihood of recording from the occipital lobe (versus the cerebellum, for example) based on the neuroanatomy of infants at this age. ${ }^{21}$

Once the probes were satisfactorily placed, signal quality for each channel was assessed using TechEn data acquisition software. Visual inspection determined whether each channel was conveying heartbeat information (a sign of good optical contact) and was detecting light at a level that was neither too high (indicating sensor saturation) nor too low. Adjustments to the probe placement and fit to the infant's head were adjusted until either all of the channels indicated good optical placement or further adjustment of the cap would have resulted in infant discomfort (either due to amount of time spent changing locations of the cap or what would have resulted in too much pressure on the infant's head). While good optical contact was experienced overall, there was spatial asymmetry as to which channels tended to have poorer optical contact. Specifically, with the design of the probe, some channels were more difficult to obtain sufficient optical contact than others. The long channels in the center of the holder (channels 8 and 7, Fig. 2) had a tendency toward worst optical contact because the design of the headband disproportionately applied pressure to the left and right of the probe holder and not the center. In addition, the short channels extended beyond the main band of the holder, which made good optical contact more difficult to achieve (channels 1, 6, 9, and 14, Fig. 2). However, despite these systematic tendencies of the probe holder, generally good optical contact was achieved across the channels and across infants who participated in the experiment.

Infants then watched an experimental video to present visual and auditory stimulation while we recorded hemodynamic changes in the occipital lobe (visual cortex). The visual stimuli were eight smiling faces from the NimStim database, ${ }^{22}$ see Fig. 4. The audio stimuli were eight common words familiar

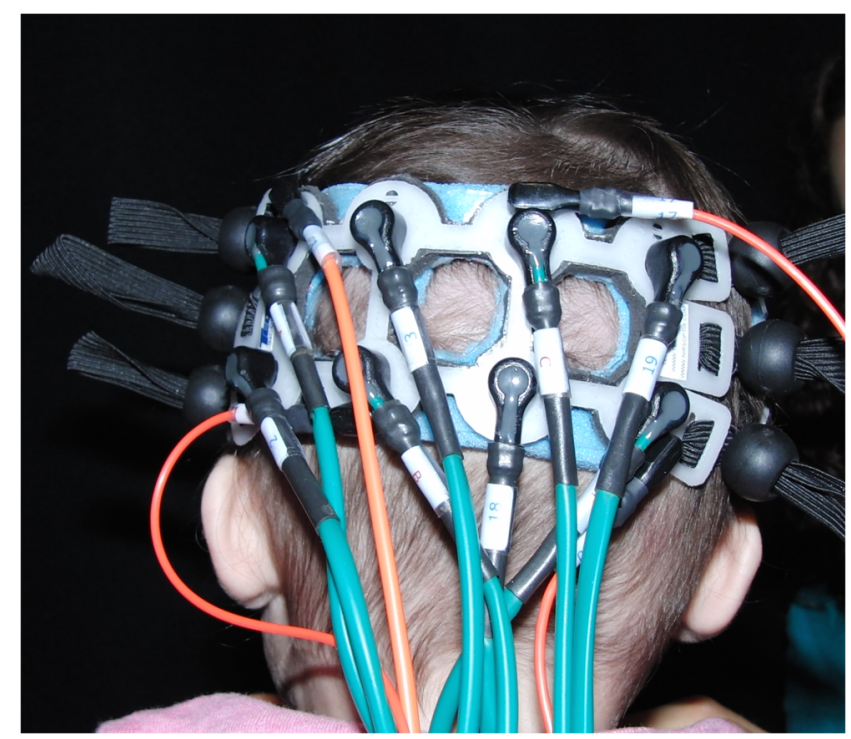

Fig. 3 Picture of probe location for infants in the current study. Probe holder was placed such that the lower row of long channels was at or slightly above the inion. Thus, the recordings were either at the inion or above it. This placement ensured recordings over the occipital lobe and not the cerebellum.

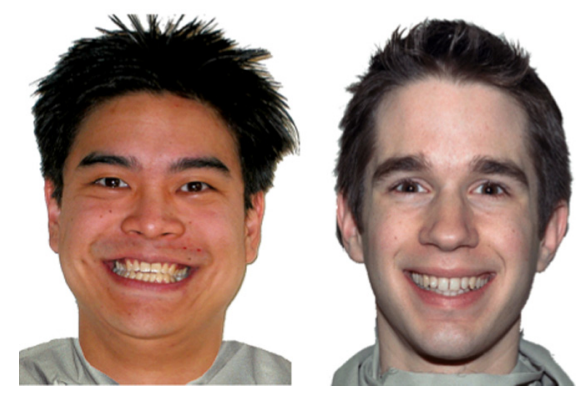

Fig. 4 Eight visual stimuli presented to infants were part of the NumStim database REF. The stimulus set included models 02,10 , $12,19,22,28,43$, and 45. Two sample models depicted. Visual stimuli were selected to be maximally visually distinct while being engaging to infants to encourage longer looking and more trials for data analysis.

to infants (apple, baby, bottle, blanket, cookie, diaper, doggie, story). The stimuli were selected to be clearly differentiable at a basic sensory level and have been found in previous studies to elicit robust auditory and visual responses (temporal and occipital cortex, respectively), but yet are engaging for infants and will encourage longer looking, which results in more trials for data analysis and trials with fewer motion artifacts. ${ }^{23}$ All stimuli had a stimulus onset asynchrony of $1 \mathrm{~s}$. The interstimulus interval (ISI) for visual stimuli was always. $25 \mathrm{~s}$. The ISI for audio stimuli ranged from 0.2 to $0.3 \mathrm{~s}$ as the duration of the auditory stimuli ranged from 0.7 to $0.8 \mathrm{~s}$.

Visual stimuli were presented in one of two block types: visual repeated blocks presented one of the eight faces eight times, and visual variable blocks presented all eight faces in shuffled order (i.e., random without replacement). While this experimental manipulation was originally designed to elicit visual repetition suppression, ${ }^{25}$ a number of studies have found that visual repetition suppression is, at best, an extremely subtle effect in infants and, at worst, not present in the occipital cortex during infancy. ${ }^{23}$ As a null effect of repetition suppression of visual stimuli in infants of this age using this protocol as a replication of previous and ongoing work, the current paper focuses on the comparison of auditory and visual stimulation in the occipital lobe rather than any differences in occipital lobe activation for the two types of visual stimulation. The auditory stimuli were always presented in auditory variable blocks (i.e., with all eight auditory stimuli presented in shuffled order).

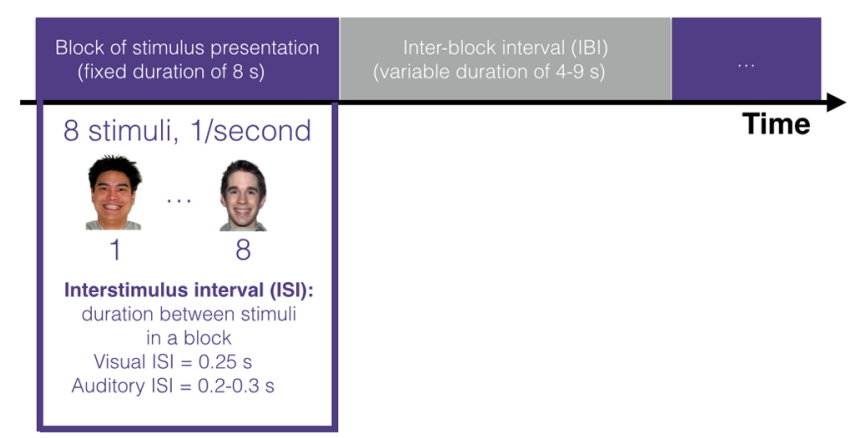

Fig. 5 Depiction of the experimental paradigm. Infants viewed blocks consisting of eight stimulus presentations each. These blocks lasted $8 \mathrm{~s}$ in total. In between blocks, there was an IBI, which had a variable length of 4 to $9 \mathrm{~s}$ to allow the hemodynamic response to return to baseline. $^{24}$ 
In between blocks, a baseline video was presented with dim fireworks on the screen and soft music. Note that under ideal circumstances, the baseline should contain neither auditory nor visual stimuli. However, it is not possible to maintain infants' attention and avoid their tendency to become fussy in the absence of any stimulation. Thus, the low-salience fireworks and music displays served as a minimal interblock baseline. Infants could watch a total of 16 visual blocks ( 8 repeated or one for each visual stimulus; eight variable) and 8 auditory blocks (all variable). Blocks were presented such that a visual repeated, visual variable, and auditory variable block were presented in a shuffled order before moving onto another shuffled order of these three block types. This counterbalancing ensured an even distribution of block types throughout the experiment. Stimuli were presented until an infant became consistently inattentive.

To control for cross-modal (auditory-visual) activation, during the audio blocks, the fireworks video continued to play on the screen, and during the visual blocks, the music continued to play in the background. Thus, all blocks contained cross-modal sensory input with the cross-modal input being the same as that employed in the baseline (e.g., for the visual blocks, the same calm, soft music as the baseline was played).

On average, infants included in the fNIRS analyses watched 5.7 auditory variable blocks $(\min =3, \max =8)$ and 6 visual variable blocks $(\min =3, \max =8)$. Infants watched an average of 17.6 blocks (including the visual repeated blocks, which averaged 5.9 blocks). No infants were excluded for not watching the video for a sufficient amount of time.

\subsection{Preprocessing}

FNIRS recordings were collected at $50 \mathrm{~Hz}$. The raw data (saved as *.nirs files) were preprocessed using HOMER2 (Homer2_UI, v1.5). ${ }^{26}$ The standard or default preprocessing method as specified in HOMER2 was followed to conform to related findings in the field. First, the "raw intensity data is normalized to provide a relative (percent) change by dividing the mean of the data" (HomER 1.0 manual), and then the signal is low-pass filtered (cutoff $3 \mathrm{~Hz}$ ) to remove high-frequency noise such as cardiac signals. Then, changes in optical density are calculated for each wavelength. Finally, the modified Beer-Lambert law is used to determine the changes (delta) concentration of oxygenated and deoxygenated hemoglobin for each channel (the procResult.dc output variable was used for subsequent analyses; as HOMER1 and HOMER2 have identical preprocessing methods for the options that we have selected, see the HOMER 1 Users Guide for full details). Subsequent analyses were conducted in MATLAB (version R2013a) with custom analysis scripts.

A notable difference between this preprocessing pipeline and what our group has employed in the past ${ }^{27}$ is the removal of a principle component analysis (PCA) offered by HOMER to remove motion artifacts. This step is intended to identify the most prominent component in the NIRS recordings, which is likely attributable to motion, and remove it. However, this preprocessing step is intended to be applied to the long channel information only. With the presence of both short and long channel, we hypothesize that the removal of the first component from the PCA analysis might include systematic vasculature, but in a way that is not easily quantified using our methods below. Indeed, we found that including this PCA step in the preprocessing removed surface vasculature signals present in the short and long channels, and, as anticipated, this early removal of surface vasculature information through PCA markedly reduced the changes in the signal when short-channel information was removed from the long channels (reported in Sec. 3). This unanticipated finding suggests that another method for removing surface vasculature signals is to apply PCA to the combination of short and long channels and remove the first principle component, which will remove a large portion of the signals from the surface vasculature (though presumably only global surface vasculature signals; see later findings for differences in global and local surface vasculature). Future work would be needed to validate this method and determine whether local and/or global vascular information is removed through this method. For the current study, we have removed this standard preprocessing step. However, given that this is one of the standard ways to remove motion from recordings, it is important to note that there is no clear evidence of motion in visual inspection of the signal (e.g., after filtering and before averaging across block types per infant or after averaging per block type per infant), or evidence that our results are contaminated by motion.

\section{Removal of Short-Channel Information from Long Channels}

Each long channel (channels 2 through 5, 7, 8, and 10 through 13, depicted in Fig. 2) was subjected to the removal of short-channel information in three different variations in order to determine whether long channels in infant NIRS recordings are contaminated by local and/or global surface vasculature signals.

\subsection{Removal of the Closest Short Channel}

Using the closest short channel allows for the identification and removal of surface vasculature signals occurring in the immediate anatomical area (i.e., local surface vascular signals) to the long channel in question as well as broadly distributed changes in surface vasculature (i.e., global surface vasculature signals, e.g., long channels 2 and 3 have the information from channel 1 removed).

\subsection{Removal of a Far Short Channel}

To elucidate the specific contribution of surface vasculature in the local region of a long channel versus the contribution of more distributed or global surface vascular signals that will be recorded by short channels located distally from the long channel, we compare the removal of a far short channel to the removal of the closest short channel. This contrast will reveal the relative contributions of local versus global surface vasculature to the long channel recordings (e.g., long channels 2 and 3 would have information from channel 9 removed). The far short channel was chosen to be a consistent distance from each long channel and in the opposite hemisphere.

\subsection{Bootstrapped Short-Channel Information}

In order to establish a baseline for comparison, we created a bootstrapped baseline by temporally shuffling the signal from the closest short channel and removing this shuffled signal from the corresponding long channels. We employ a temporal shuffling method because it maintains the distributional properties of the short channel while allowing us to determine how much the removal of short-channel information depends on both the short and the long channels recording the same vascular events (e.g., the current change of blood pressure). The shuffled 
short-channel information is a discontinuous signal with successive time points likely being drawn from disparate time points in the original signal. Importantly, temporal shuffling ensures that any changes in the bootstrapped signal are not arising from the same vascular events as the long channel. Thus, removing shuffled short-channel information allows us to determine the likelihood that removing short-channel information will change the long channel if the short channel is not recording from the same vascular events. To illustrate, if a given baby contributed fNIRS recordings from time points 1 to $N$, the short-channel signal was randomly shuffled without replacement so all individual time points ( 1 to $N$ ) were still included but were presented in a different (random) order. This shuffled shortchannel information is then removed from the long channels. To obtain a bootstrapped baseline of signal change, we repeat this shuffling and removing method 1000 times. For example, long channels 2 and 3 would have the temporally shuffled signal from channel 1 removed. This is repeated 1000 times (1000 different shuffles of channel 1).

Following the work by Berger and colleagues, ${ }^{10,11,15,28}$ we employed the following equations to remove the short-channel information:

$$
\begin{aligned}
\alpha= & \text { ShortChannel }^{\top} \cdot \text { LongChannel } \\
& \div\left(\text { ShortChannel }^{\top} \cdot \text { ShortChannel }\right)
\end{aligned}
$$

LongChannel' $=$ LongChannel $-\alpha \cdot$ ShortChannel.

Note that equations are applied on oxygenated hemoglobin after filtering has taken place. Variables represent column vectors with the length equal to the number of time points recorded for a given subject and $\mathrm{T}$ represents the transpose operation.

Our first goal was to determine how much of the signal from the long channels changed in these three different types of removal schemes: bootstrapped baseline, far short channel, and closest short channel. To this end, we compared LongChannel' from Eq. (1) with the original LongChannel and determined

1. the average absolute change in signal when the shortchannel information was removed, |LongChannel' LongChannel|

2. how many data points were changed beyond 2 standard deviations (determined for each original long channel). The number of points is presented as a percentage because all infants contributed different amounts of fNIRS recordings.

The change in signal was considered starting at the first sample taken during the start of stimulus presentation and considered until the end of recording. Signal changes are considered for oxygenated $\mathrm{Hb}$ as this is the most common NIRS measure reported in the infant literature. ${ }^{1}$

Initial inspection of the data revealed that one infant (baby 1) was a clear outlier in two respects. First, despite using the same preprocessing methods, the baby's averaged signal was consistently below baseline and the raw signal exhibited unexplained and dramatic jumps. These two characteristics strongly suggest poor signal quality for this infant. Second, the removal of shortchannel information resulted in signal changes that are more than an order of magnitude greater than the other infants for both the magnitude of signal changes and the number of time points changed. Short-channel removal dramatically changed the data of this infant such that they would not be identified as an outlier after short-channel removal. However, given the poor signal quality in this infant's data, it is difficult to interpret this substantial change in signal quality after short-channel removal. As a very clear outlier, we removed the data from this infant from all subsequent analyses (male, age $=6.9$ months, Caucasian, nonHispanic), though all results are qualitatively identical with and without the inclusion of this infant.

Having obtained our final sample, both the magnitude of the signal change and the percentage of data points significantly changed are depicted across the three types of short-channel information (bootstrap, far, and closest), in Figs. 6 and 8, respectively.

\subsection{What Is the Average Change to the Long- Channel Signal After Short-Channel Removal?}

Figure 6 shows the average magnitude of signal change across the three different short channels (bootstrap, far, and closest) revealing clear differences across short-channel type. Similarly, Fig. 7 presents scatterplots for the three different short channels for each infant [Fig. 7(a)] and channel [Fig. 7(b)]. This scatterplot also indicates clear differences between short-channel types across the same channels and infants.

To confirm that removing far and closest short-channel information changes the signal more than the bootstrapped short channel, while controlling for variability between infants and channels, we constructed a linear mixed effects model. ${ }^{29,30}$ The model predicted MeanSignalChange from the following equation: ShortChannelType + (1l Infant) + (1l Channel) with the bootstrapped short-channel set as the intercept $[\beta=1.433 \mathrm{e}-04, t(25)=3.613, \quad p=0.00132]$. We find a significant effect of the far channel $[\beta=1.342 \mathrm{e}-04, t(430.2)=$ $3.839, \quad p=0.000142]$ and the closest channel $[\beta=$ $2.167 \mathrm{e}-04, t(430.2)=6.20, p<0.00001]$. This model indicates that the far and closest short channels are significant predictors of the magnitude of the raw signal changed beyond the bootstrap short channel. Thus, compared to a bootstrapped

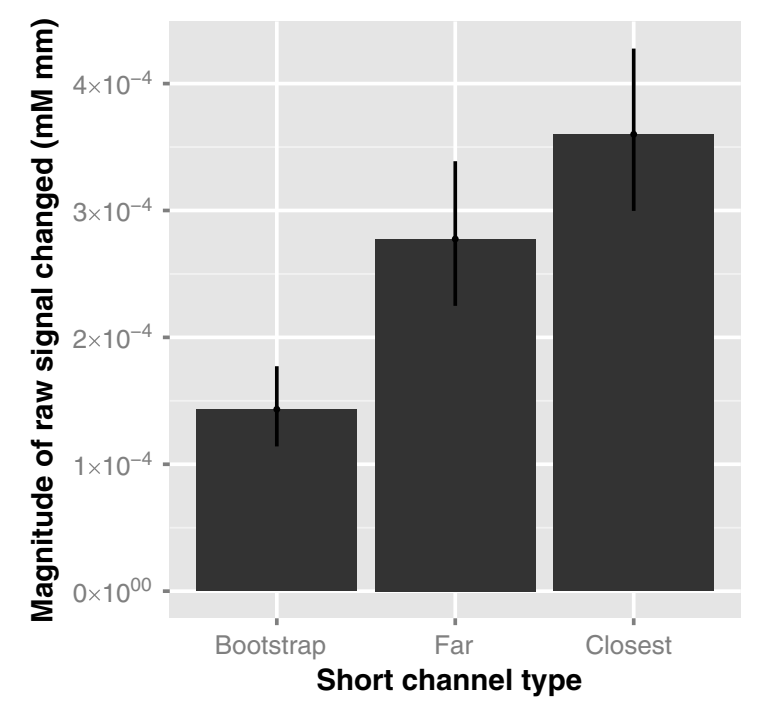

Fig. 6 Average absolute signal change for three different versions of short-channel removal described in Sec. 3. The average absolute value of the signal is $1.1867 \times 10^{-3}$ (standard deviation of $\left.8.9797 \times 10^{-4}\right)$. Error bars are bootstrapped confidence intervals around the mean. 


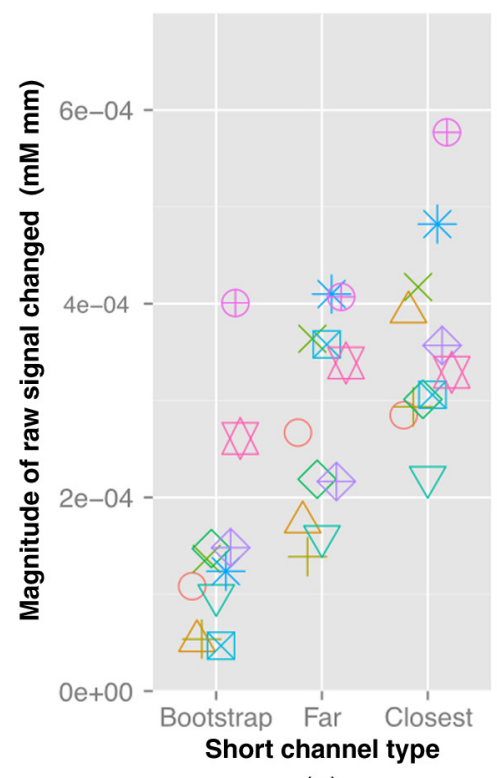

(a)

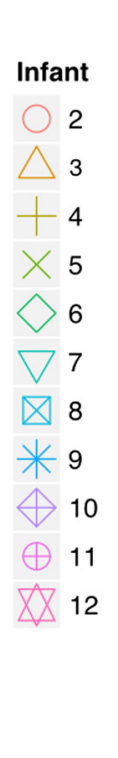

Fig. 7 Average absolute signal change (a) per infant and (b) per long channel for three different versions of short-channel removal described in Sec. 3.

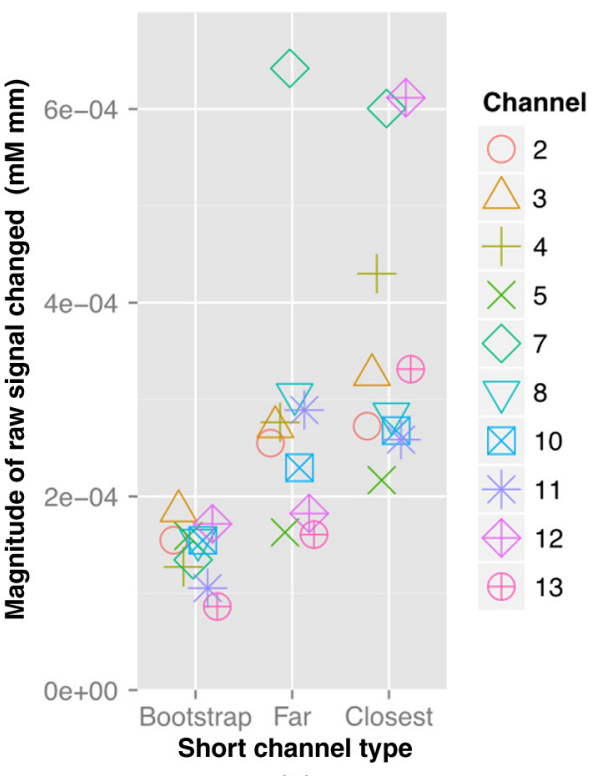

(b) baseline, removing information from either the far or the closest short channels significantly changes the long-channel signals.

To investigate whether there are any differences between the far and closest short channels, we ran the same linear mixed effects model but reordering the levels of the short-channel factor so that the closest channel is the intercept. Here we find a significant contribution of the far short channel $[\beta=-8.251 \mathrm{e}-05, t(430.2)=-2.360, p=0.0187$; intercept: $\beta=3.600 \mathrm{e}-04, t(25)=9.077, p<0.00001]$. Thus, even after controlling for any variability in infants and channels, we find that there is a difference in the magnitude of the signal change between the far and closest short channel, suggesting that there are both local and global surface vasculature contributions to long-channel recordings.

\subsection{What Is the Proportion of Data Points Significantly Changed after Short-Channel Removal?}

As a complement to the analysis of mean signal change in Sec. 3.1, here we investigate what percentage of data points are significantly changed when short-channel information is removed. Increases in mean signal change can arise from either large changes to a small number of points or small changes to a large number of points. Here, we investigate the number of data points that exhibit large changes after the removal of short-channel information. We quantify large changes as being changes equal to or greater than 2 standard deviations (SD) for that channel from the original value for that data point. Since the task was infant-controlled (i.e., infants watched for different amounts of time), we express the number of points as a percentage of the total signal for that infant starting from the presentation of the stimulus to the end of the experiment.

This analysis is entirely convergent with the analysis of mean signal change: removing far and closest short-channel information changes the signal more than the bootstrapped baseline and there is additional benefit to removing the closest short-channel information. As illustrated in Fig. 8, the bootstrap analysis resulted in zero significantly changed data points. There were no points that changed at least $2 \mathrm{SD}$, suggesting that the mean signal changes reported in Sec. 3.1 are derived from small changes to a relatively large number of data points. Similar to the analysis of mean signal change, we find that the removal of far and closest short-channel information results in more changes to the signal than the bootstrap control demonstrating (1) that the short-channel information of the far and closest short channels is beyond a baseline and (2) that even the far short channel contains more information than a baseline, suggesting the presence of broadly distributed vascular information embedded in the long channels. We also find differences in the percentage of data points changed between the far and closest short channels, where the closest short channel results in

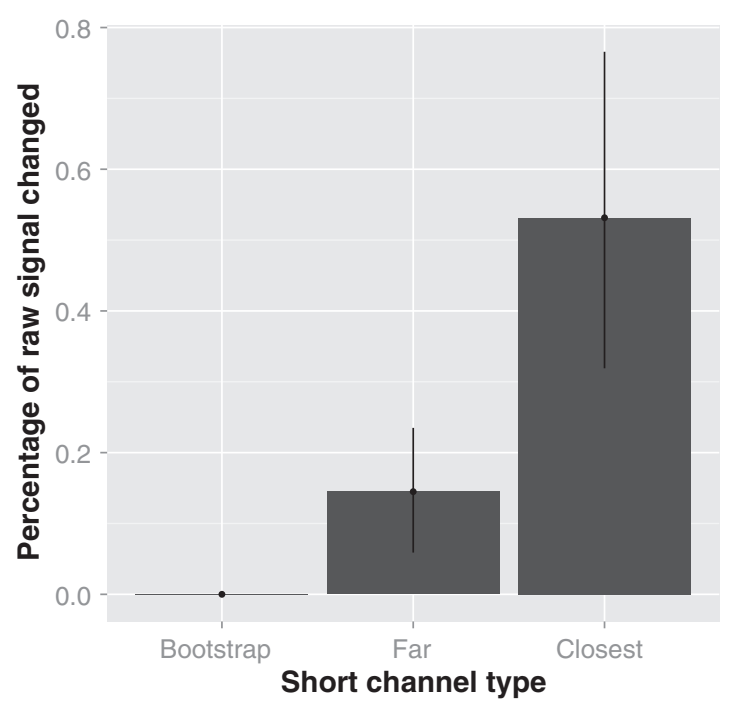

Fig. 8 Percentage of data points changed beyond 2 standard deviations for the channel across the three different variations of shortchannel removal described in Sec. 3. 

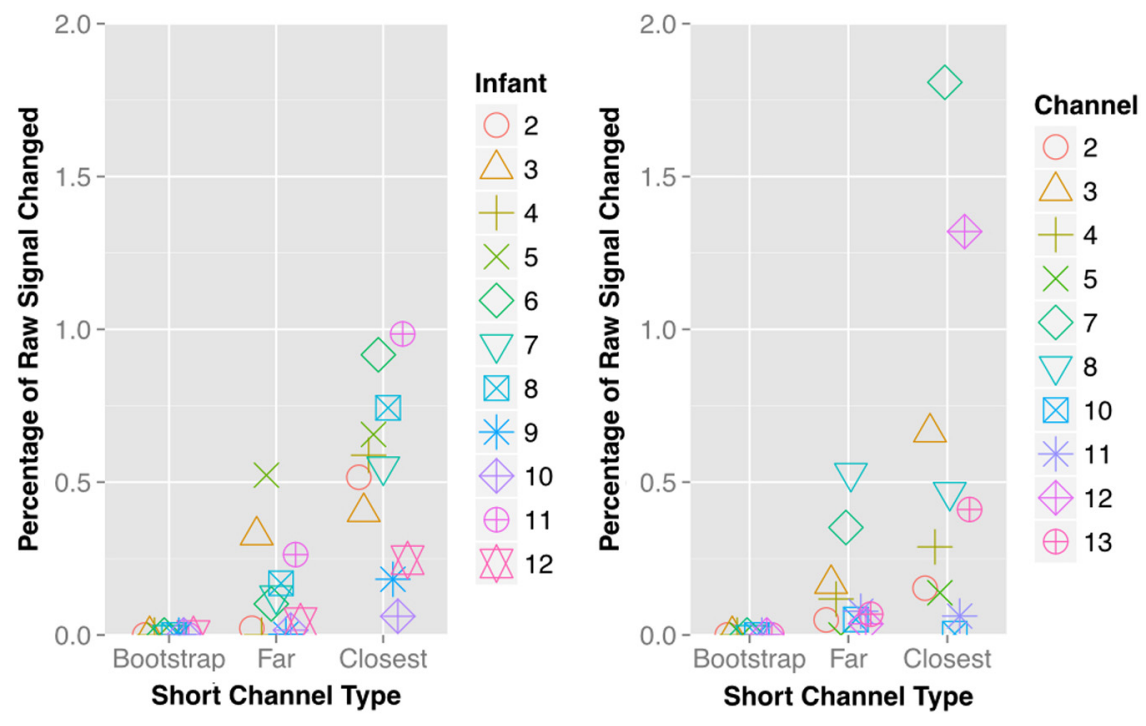

Fig. 9 Percentage of points changed beyond 2 standard deviations (a) per infant and (b) per long channel for three different variations of short-channel removal described in Sec. 3.

more data points being changed than the far short channel. Figure 9 presents a scatterplot of the percentage of data points changed by infant [Fig. 9(a)] and channel [Fig. 9(b)] and also suggests differences across short-channel type.

To assess whether there are differences between removal of far and closest short channel, the data were modeled with a mixed effects logistic regression ${ }^{29}$ including random effects for infant and channel. We excluded the bootstrap variation because the logistic regression cannot accommodate a factor with all zeros, and it is clear that there are statistically significant differences between the bootstrap and the far and closest short channel.) In the model, we predicted the number of data points changed based on the type of short-channel information removed, while controlling for differences across infants and channels (each considered as a random factor. In other words, the model's equation is Number of Points Changed/ Total Points $=$ ShortChannelType $+(1$ Infant $)+(1 \mid$ Channel $)$ with the far short-channel set as the intercept $(\beta=-7.41$, $Z=-16.05, p<0.00001)$. We find that including the closest short channel significantly predicts some of the variance $(\beta=1.31, Z=55.72, p<0.00001)$. Thus, this model reveals that there are differences between the two types of short channels, with the closest short channel changing more data points than the far short channel.

\section{Effects of Removing Short-Channel Information on Neural Activity Outcomes}

Having determined how different types of short-channel removal affect the oxygenated hemoglobin signal, these signals (with and without short-channel information removed) were then submitted to our standard analysis in order to determine how the removal of short-channel information affects our outcome measures.

These analyses consisted of determining the average and variability (standard error) in the concentration changes of oxygenated hemoglobin for all long channels and for all included infants to the three different stimulus block types: auditory, visual repeated, and visual variable. As discussed earlier, we will focus on the comparison of visual (repeated) and auditory as the current work confirms the lack of widespread differences in activity to visual repetition (versus variability) in the occipital lobe in infancy. ${ }^{23}$ Any trials where the infant did not view the stimuli for $14.5 \mathrm{~s}$ after the start of the trial were dropped; this length of time represents the average time from the start of the trial to the end of the jittered interblock interval (IBI, block duration is $8 \mathrm{~s}$ with an average IBI of $6.5 \mathrm{~s}$ and a range of 4 to $9 \mathrm{~s}$, see Ref. 24 for validation of this IBI).

Figure 10 presents responses for the long channels without any short-channel information removed. Significant responses in the occipital lobe to visual stimuli of infants of this age have been well established as well as selectivity of this region to visual stimuli. ${ }^{23,27,31-33}$ Consistent with this previous work, there is a significant response in the occipital lobe to the presentation of visual stimuli. Following standard analysis procedures, we compared responses within an a priori time window (6 to

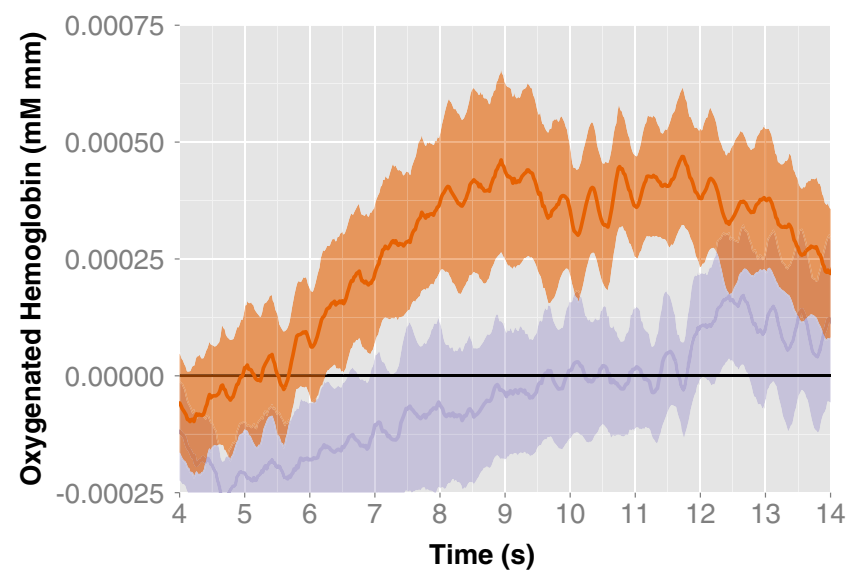

Fig. 10 Changes in the signal (oxyHb) for long channels (before removal of short-channel signals) to the auditory block (purple or light grey) and the uniform visual block (orange or dark grey). The long channels independently reveal a significant cortical response and a visual selectivity of the occipital lobe to visual stimuli. Signals were averaged across all the long channels as the probe configuration was designed so all channels record from the occipital lobe (i.e., the ROI) and the stimuli were designed to produce broad responses within this lobe. 


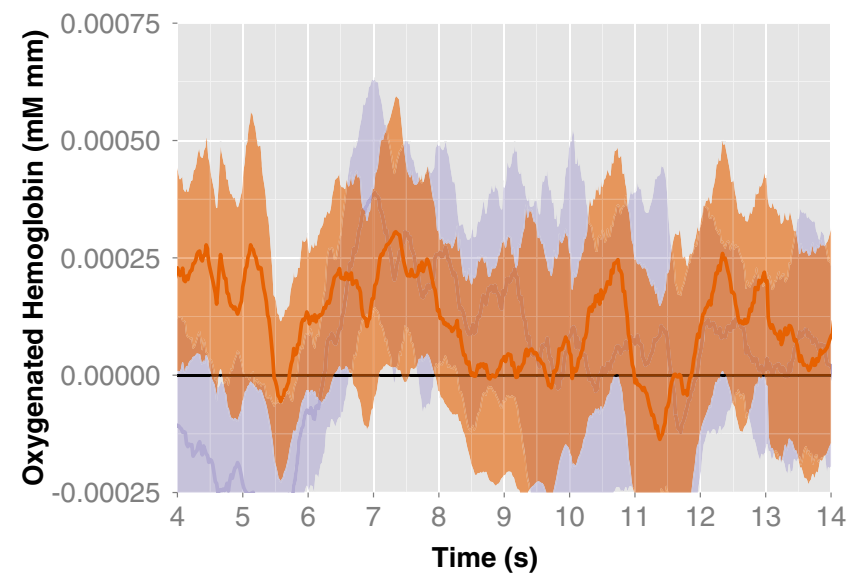

Fig. 11 Changes in the signal (oxyHb) for the short channels to the auditory block (purple or light grey) and the uniform visual block (orange or dark grey). Unlike the long channels, the short channels reveal no significant cortical response nor visual selectivity of the occipital lobe to visual stimuli. Signals were averaged across all the short channels.

$14.5 \mathrm{~s}$ after stimulus onset) where the hemodynamic response typically occurs in infants in response to these blocked stimuli compared to chance level (zero). We find a response above baseline in the visual condition $[t(10)=2.4620, p=0.0336]$ but not in the auditory condition $[t(10)=-0.052, p=0.9590]$ and a marginally significant difference in response between the two conditions $[t(10)=-2.1346, p=0.0586]$. Thus, consistent with previous work, we find robust visual responses in the occipital lobe of infants, and some evidence for visual selectivity in this region of the infant brain.

We now consider visual responses only and compare the long-channel responses before the removal of short-channel information and after the removal of two types of short-channel information: closest short channel and far short channel. Figure 12 presents hemodynamic responses (oxyHb) averaged for the long channels before (orange) and after (blue, green)

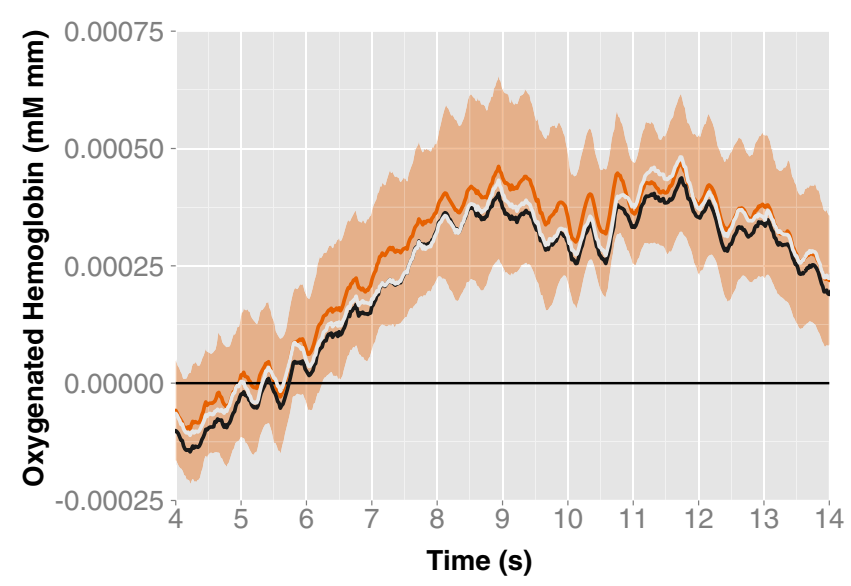

Fig. 12 Changes in oxygenated $\mathrm{Hb}$ in response to visual blocks for the long channels. Original long channel signals are presented in orange or dark grey (same as in Fig. 10). Black and light grey responses are the signal when short-channel information is removed: Far short channel removed is depicted in black and closest short channel removed is depicted in light grey. Signals were averaged across all the long channels as the probe configuration was designed so all channels record from the occipital lobe (i.e., the ROI) and the stimuli were designed to produce broad responses within this lobe. removing short-channel information. Removing the closest short-channel information (green) and removing the far shortchannel information (dark blue) result in minimal differences in the hemodynamic response. Specifically, we continue to see significant responses in these channels to visual stimuli ( $t^{\prime} s>2.78, p ' s<0.02$ ), but the response is no more robust after short-channel information is removed.

Considering the auditory response only, we compare responses before and after the removal of short-channel information. Figure 13 presents the averaged hemodynamic responses and reveals that, similar to the visual responses, while there are changes in the responses, we do not find a significant response to auditory information above baseline. Statistical tests also confirm that there is no significant hemodynamic response in the occipital lobe to auditory stimuli $(t ' s<0.6, p$ 's $>0.5)$.

Finally, comparing the visual and auditory responses, we continue to find marginally significant differences between these conditions ( $t$ 's $>-2, p$ 's $<0.07)$. Thus, we do not find that removing short-channel information boosts the difference between the auditory and visual responses to significance.

In sum, considering how the removal of short-channel information affects stimulus-evoked responses yields a different picture. While we found that removing short-channel information significantly alters fNIRS signals in infants, we do not find that these signal differences affect the outcome of standard analyses. The overall pattern of responses in the long channels, without short-channel information removed, indicated a significant visual response, no auditory response, and marginal visual selectivity. This pattern was qualitatively unchanged after short-channel information is removed from either the closest or far short channel.

There are two notable conclusions of this analysis and some qualifications. First, we find that while there is significant signal change resulting from short-channel removal, these changes do not substantially affect outcomes from higher-level statistical analyses or conclusions from the experiment. Second, while

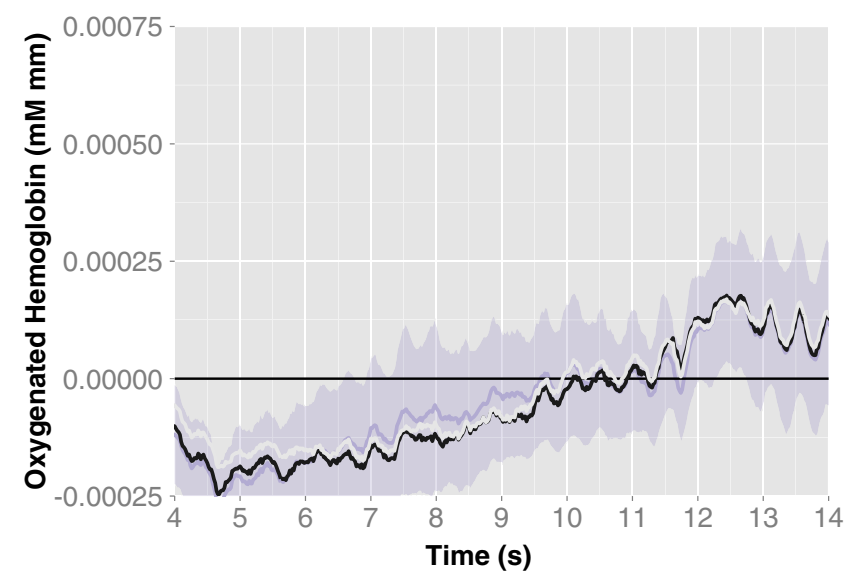

Fig. 13 Changes in oxygenated $\mathrm{Hb}$ in response to auditory blocks for the long channels. Purple or medium grey response is before the removal of short-channel signals (same as in Fig. 10). Black and light grey responses present the signal after short-channel information is removed: Far short channel removed is depicted in black and closest short channel removed is depicted in light grey. Signals were averaged across all the long channels as the probe configuration was designed so all channels record from the occipital lobe (i.e., the $\mathrm{ROI}$ ) and the stimuli were designed to produce broad responses within this lobe. 
we found that the magnitude of signal change and the proportion of data points changed is higher for the closest short channel, we do not find that this translates to better signal quality with regards to the use of these signals for inference about hemodynamic responses to stimulus presentation. However, this lack of difference could result from the general lack of signal change rather than a result of short-channel removal. Moreover, it is possible that the enhanced effects of removing short-channel information only arise when there is not a robust hemodynamic response to begin with, or perhaps under different experimental conditions (e.g., when infants are viewing particularly salient stimuli that may cause blood pressure changes).

\section{Does a $1 \mathrm{~cm}$ Short Channel in Infants Show Evidence of Cortical Activity?}

Our short-channel information is obtained using $10 \mathrm{~mm}$ emitterdetector channels. This channel separation or larger is standard (e.g., 12, $13 \mathrm{~mm}$ ). However, recent work on adults has suggested that $13 \mathrm{~mm}$ channels contain cortical information and channels $<10 \mathrm{~mm}$ (e.g., $6 \mathrm{~mm}$ ) are better for isolating surface vasculature signals and not detecting task-related hemodynamic changes presumably coming from cortical sources. ${ }^{28}$ See also simulations by Strangman and colleagues ${ }^{34}$ suggesting $10 \mathrm{~mm}$ channels sample from some gray matter but $5 \mathrm{~mm}$ channels do not. This work raises a concern that our short channel might be too long for its purpose. Moreover, this problem may be exacerbated because we are recording from infants who have systematically thinner skulls than adults. ${ }^{35}$ While future work is certainly needed to establish the best distance for short-channel recordings in infants, we sought to establish that $10 \mathrm{~mm}$ channels do not contain a large amount of cortical activity that would be removed.

From previous results reported in this paper, it is clear that our pattern of results does not suggest that we are removing cortical activity when we remove the short-channel information. Without removing short-channel information, our long channel demonstrably contains cortical, stimulus-evoked hemodynamic changes (see Sec. 4 and Fig. 10). If the short channels contained even these cortically produced hemodynamic changes, when we removed the short-channel information, we should see reductions to the averaged, stimulus-evoked waveforms for the long channels. Section 4 demonstrates that we do not see large changes in the stimulus-evoked responses and, indeed, maintain a significant response to visual stimulation in the occipital channels (Fig. 12). This pattern of results suggests that our short-channel information does not contain cortically based hemodynamic activity.

However, a more direct way to confirm that our short channels do not contain cortical activity is to subject our short channels to the same analyses conducted on the long channels (i.e., see if there are significant responses to the stimuli). Figure 11 presents the averaged short-channel responses for the auditory and visual blocks (see Fig. 10 for the corresponding responses from the long channels to note the differences). Using the same analytic methods as the long channels, we find that there are no significant responses in the short channels to any of the three stimulus blocks $(p>0.35)$. Specifically, in the visual blocks reported in Sec. 4 and Fig. 10, the short channels exhibit no response: $t(10)=0.5066, p=0.6234$.

Future work will be needed to determine the length of short channel that is ideal to isolate surface vasculature activity in infants. The current work suggests that a $10 \mathrm{~mm}$ short channel does not contain a significant proportion of cortical activity. This finding is similar to previous work by Taga and colleagues, ${ }^{20}$ who investigated both source-detector separation $(1,2,3$, $4 \mathrm{~cm}$ ) and light intensity in infants on average two months younger than those reported in the current study. Taga and colleagues determined that the $10 \mathrm{~mm}$ channels did not have robust evidence of cortically based changes to the hemodynamic response. Instead, Taga and colleagues found that the optimum distance was $2 \mathrm{~cm}$. Similarly, the McDonnell Infant Methodology Consortium for NIRS (Ref. 6) underwent a systematic investigation of the optimum source-detector distance for infants and found that somewhere between 2 and $3 \mathrm{~cm}$ was the optimum, which confirmed results from Ref. 20 though distances shorter than $2 \mathrm{~cm}$ were not systematically investigated. Thus, while the optimum distance for short-channel measurements of the surface vasculature in infants has yet to be determined, current and past work suggests that $10 \mathrm{~mm}$ does not obviously record hemodynamic changes arising from cortical activity but comparisons to smaller channel separations are needed.

\section{Conclusions and Discussion}

The present study investigated the signals obtained from short fNIRS channels in human infants. Specifically, we investigated whether short-channel signals could successfully remove signals arising from surface vasculature in human infants, and if so, whether the removal of short-channel information could result in changes in the statistical inferences made about fNIRS responses to different stimulus conditions (i.e., had an empirical effect). In addition, we examined whether there is evidence for local variations in surface vasculature signals in infants and whether removing a local short channel has a benefit compared to removing information from a far short channel recording more global surface vasculature signals.

Overall, we found that removing short-channel information, using methods employed by Berger and colleagues, ${ }^{10,11,15,28}$ does significantly change the signal in the long channel. We determined both the average signal change as well as the number of data points significantly changed (i.e., changed by more than 2 standard deviations) and then compared both measures to a bootstrapped baseline (see Sec. 3 for details). We find evidence for both global and local signals arising from the surface vasculature. We removed short-channel information for both the closest short channel and a far short channel to estimate the contributions of local versus global surface vasculature signals. The closest short channel presumably includes any local as well as any global surface vascular signals, whereas the far channel (located on the other hemisphere and $6 \mathrm{~cm}$ away from the light source for the fNIRS channel) will record only global surface vascular signals. Compared to the bootstrapped baseline, we find that removing information from both the closest and far short channels significantly changes the fNIRS channel. However, we find an additional benefit of removing the closest short channel. These findings converge with reports that local measurements of surface vascular signals yield the greatest changes in the long-channel signals. ${ }^{14,15}$

However, despite significant changes in the long-channel signal, we did not find that removing short-channel information resulted in any differences in the statistical inferences made from the experiment. Specifically, we found evidence of a robust and selective visual response without the removal of short-channel information and continued to find the same response after short-channel removal (either the closest or far short channels). 
Thus, we do not find that removing the short-channel information yields more effective or even different statistical inference.

There are a number of reasons why we might not see a significant difference as a result of removing short-channel information and future work will be needed to disentangle these possibilities. One possibility for not seeing an effect is that the neurovascular signals were robust enough in the long channels that any effect of surface vasculature was small. In other words, the presence of a robust and selective response to begin with did not require the additional benefit of removing systematic vasculature by means of the short channel. We had included auditory presentation as a circumstance in which we would not expect a neurovascular response and, thus, might benefit from short-channel removal, and in that instance as well, we find no difference in statistical inferences before and after short-channel removal. This result is similar to recent work by Yücel and colleagues. ${ }^{16}$ This excellent recent work examined the effects of short-channel removal in the adult motor cortex during a finger tapping task versus presentation of a painful electric stimulus. Importantly, the work examined the effects of short-channel removal on the results of statistical analysis. This group also found that removal of short channel did not result in changes in statistical result for the finger tapping task; however, they did find effects in the painful stimulus condition. A major difference between these conditions is that the nature of the latter, but not the former, stimulus is more likely to cause arousal-based circulatory responses that will result in increased activity in the surface vasculature. This result suggests that short-channel information removal might be most beneficial under circumstances of heightened arousal and not more cognitive-based tasks as was investigated in the current study. Thus, another possibility why we did not find significant effects of short-channel removal is that the current stimuli investigated do not give rise to significant signals in the surface vasculature. If this were the case, the need for short-channel recordings in standard developmental cognitive neuroscience studies is less justified as surface vasculature effects are subtle, may be removed with standard preprocessing procedures (e.g., filtering to remove heart rate signals), and, in the current case, do not bias to statistical inferences drawn from the study.

Yet another possibility is that the removal of short-channel information is less beneficial in infants than in adults. As reviewed in Sec. 1, there are significant developmental differences in the circulatory system, the tissues surrounding the brain (e.g., the skull) as well as neurovascular coupling. Given these developmental differences, it is possible that the circulatory signals in the scalp and the skull contribute less to fNIRS signals in infants than adults, and thus, the confounding fluctuations in the scalp are negligible in infants. Thus, the lack of significant differences in removing shortchannel information in infants might be reflective of differences in the composition of the long-channel signal (i. e., how much of the signal is coming from the surface vasculature) between infants and adults.

One final possibility is that the current methods, which applied short-channel removal broadly across all channels and used only standard measures for optical contact available with the recording device and visual inspection for obvious issues with signal quality (see the excluded infant), are too blunt to reap the benefits of short-channel removal. It is important to note that we do see differences in the benefit for shortchannel removal across channels suggesting that there might be differences in either recording quality or location of surface vasculature signals. However, even after controlling for the effects of channels, we still see evidence for signal change after shortchannel removal. Despite seeing evidence that removing shortchannel information does change the long-channel signals, it is possible that some variation of our application of these methods would be needed before short-channel removal would benefit the statistical outcomes of the experiment. However, in order for the removal of short-channel information to be broadly adopted by cognitive neuroscientists, any method will have to result in differences at the level of statistical inference in the context of standard data analysis procedures for the field. Moreover, it is important to note that we only employed one method for removing short-channel information (i.e., the method employed by Berger and colleagues ${ }^{10,11,15,28}$ ), and there are other methods that can be employed to this end (e.g., Refs. 12 and 13). It is possible that other analytic methods will reveal a different result. While not systematically explored in this paper, we did find that including PCA in our preprocessing methods (a method similar to Refs. 12 and 13) did reduce the evidence of signal change but did not change the resulting statistical tests, similar to not using PCA and including short-channel regression. Moreover, there are different methods of fNIRS analyses that might be more or less sensitive to surface vasculature signals. The current analysis sought to determine whether there is robust response within a region of interest (ROI) located over the occipital cortex to a visual stimulus and whether this response is selective to visual stimuli or whether this ROI also responds to auditory stimuli. Additionally, one might ask how precise the activation is (i.e., localization of the hemodynamic response in the cortex) and consider whether removing surface vasculature signals might increase localization (i.e., reduce the number of channels that respond to a given stimulus). It will be fruitful to explore the effects of surface vasculature signal removals on this and other types of functional analysis of fNIRS data (e.g., functional connectivity) in future work. Thus, it is not our claim that all methods of removing shortchannel info will have no impact on any type of neuroimaging analysis in infants, and we believe that future work in this vein is necessary to demonstrate whether the current results are generalizable or specific to the analyses, populations, and task employed in the current study.

Investigation of hemodynamic signals arising from surface vasculature is important for the field, and as surface vasculature signals can, in principle, cause both type I and type II statistical errors, this methodological issue has the potential to have widereaching ramifications in the studies that employ fNIRS to make statistical inferences about neural activity (e.g., developmental cognitive neuroscience). The current finding confirms the presence of surface vasculature signals in fNIRS recordings in infants (i.e., that removing short-channel information changes long fNIRS channels) but does not provide evidence that signals from the surface vasculature are biasing statistical inferences in infants in a standard experimental task to measure visual responses (i.e., that removing surface vasculature signals through this method affects statistical inferences drawn from the study).

\section{Acknowledgments}

The authors thank Holly Palmeri, Ashley Rizzeri, and all the research assistants in the Rochester Baby Lab. Thanks to TechEn for the generous use of their machine for this project 
and, in particular, to Buzz DiMartino and Bob Dewsnap for their help in this regard. Special thanks to the caregivers and babies who participated in the study. Funding for this project was provided by NIH Grants K99 HD076166-01A1 and 4R00HD076166-02, and a Canadian Institutes of Health Research postdoctoral fellowship (to L.L.E.) and NIH Grant R01 HD-37082 (to R.N.A.).

\section{References}

1. R. N. Aslin, M. Shukla, and L. L. Emberson, "Hemodynamic correlates of cognition in human infants," Annu. Rev. Psychol. 66, 349-379 (2015).

2. J. Gervain et al., "The neonate brain detects speech structure," Proc. Natl. Acad. Sci. U. S. A. 105, 14222-14227 (2008).

3. G. Taga et al., "Brain imaging in awake infants by near-infrared," Proc. Natl. Acad. Sci. U. S. A. 100(19), 10722-10727 (2003).

4. T. Wilcox et al., "Hemodynamic response to featural changes in the occipital and inferior temporal cortex in infants: a preliminary methodological exploration: paper," Dev. Sci. 11(3), 361-370 (2008).

5. R. N. Aslin and J. Mehler, "Near-infrared spectroscopy for functional studies of brain activity in human infants: promise, prospects, and challenges," J. Biomed. Opt. 10(1), 011009 (2005).

6. J. Gervain et al., "Near-infrared spectroscopy: a report from the McDonnell Infant Methodology Consortium," Dev. Cognit. Neurosci. 1, 22-46 (2011).

7. Y. Minagawa-Kawai et al., "Optical imaging of infants' neurocognitive development: recent advances and perspectives," Dev. Neurobiol. 68, 712-728 (2008).

8. R. N. Aslin, "Questioning the questions that have been asked about the infant brain using near-infrared spectroscopy," Cogn. Neuropsychol. 29(1-2), 7-33 (2012).

9. L. Gagnon et al., "Quantification of the cortical contribution to the NIRS signal over the motor cortex using concurrent NIRS-fMRI measurements," Neurolmage 59, 3933-3940 (2012).

10. R. B. Saager and A. J. Berger, "Direct characterization and removal of interfering absorption trends in two-layer turbid media," J. Opt. Soc. Am. A 22(9), 1874-1882 (2005).

11. R. Saager and A. Berger, "Measurement of layer-like hemodynamic trends in scalp and cortex: implications for physiological baseline suppression in functional near-infrared spectroscopy," J. Biomed. Opt. 13(3), 034017 (2008).

12. Q. Zhang, E. N. Brown, and G. E. Strangman, "Adaptive filtering for global interference cancellation and real-time recovery of evoked brain activity: a Monte Carlo simulation study," J. Biomed. Opt. 12(4), 044014 (2007).

13. Q. Zhang, G. E. Strangman, and G. Ganis, "Adaptive filtering to reduce global interference in non-invasive NIRS measures of brain activation: how well and when does it work?," NeuroImage 45(3), 788-794 (2009).

14. L. Gagnon et al., "Further improvement in reducing superficial contamination in NIRS using double short separation measurements," NeuroImage 85(Pt 1), 127-135 (2014).

15. R. B. Saager et al., "Two-detector corrected near infrared spectroscopy (C-NIRS) detects hemodynamic activation responses more robustly than single-detector NIRS," NeuroImage 55(4), 1679-1685 (2011).

16. M. A. Yücel et al., "Short separation regression improves statistical significance and better localizes the hemodynamic response obtained by near-infrared spectroscopy for tasks with differing autonomic responses," Neurophotonics 2(3), 035005 (2015).

17. M. S. Beauchamp et al., "The developmental trajectory of brain-scalp distance from birth through childhood: implications for functional neuroimaging," PLoS One 6, e24981 (2011).

18. J. J. Harris, C. Reynell, and D. Attwell, "The physiology of developmental changes in BOLD functional imaging signals," Dev. Cognit. Neurosci. 1, 199-216 (2011).

19. E. M. C. Hillman, "Coupling mechanism and significance of the BOLD signal: a status report," Annu. Rev. Neurosci. 37, 161-181 (2014).

20. G. Taga, F. Homae, and H. Watanabe, "Effects of source-detector distance of near infrared spectroscopy on the measurement of the cortical hemodynamic response in infants," NeuroImage 38(3), 452460 (2007).

21. P. T. Fillmore et al., "Stereotaxic MRI brain atlases for infants from 3 to 12 months of age," Dev. Neurosci. 37(6), 515-532 (2015).

22. N. Tottenham et al., "The NimStim set of facial expressions: judgments from untrained research participants," Psychiatry Res. 168, 242-249 (2009).

23. L. L. Emberson et al., "Using fNIRS to examine occipital and temporal responses to repetition in young infants: evidence of frontal cortex involvement" (under review).

24. M. M. Plichta et al., "Model-based analysis of rapid event-related functional near-infrared spectroscopy (NIRS) data: a parametric validation study," NeuroImage 35, 625-634 (2007).

25. K. Grill-Spector, R. Henson, and A. Martin, "Repetition and the brain: neural models of stimulus-specific effects," Trends Cognit. Sci. 10(1), 14-23 (2006).

26. T. J. Huppert et al., "HomER: a review of time-series analysis methods for near-infrared spectroscopy of the brain," Appl. Opt. 48(10), C280C298 (2009).

27. L. L. Emberson, J. E. Richards, and R. N. Aslin, “Top-down modulation in the infant brain: learning-induced expectations rapidly affect the sensory cortex at 6-months," Proc. Natl. Acad. Sci. U. S. A. 112(31), 95859590 (2015).

28. J. R. Goodwin, C. R. Gaudet, and A. J. Berger, "Short-channel functional near-infrared spectroscopy regressions improve when sourcedetector separation is reduced," Neurophotonics 1(1), 015002 (2014).

29. D. Bates et al., "Fitting linear mixed-effects models using lme4," J. Stat. Software 67(1), 1-48 (2015).

30. A. Kuznetsova, P. B. Brockhoff, and R. H. B. Christensen, "ImerTest: tests in linear mixed effects models," R package version 2.0-25 (2015). http://CRAN.R-project.org/package=lmerTest

31. G. Taga et al., "Hemodynamic responses to visual stimulation in occipital and frontal cortex of newborn infants: a near-infrared optical topography study," Pathophysiology 10(3-4), 277-281 (2004).

32. G. Taga and K. Asakawa, "Selectivity and localization of cortical response to auditory and visual stimulation in awake infants aged 2 to 4 months," Neurolmage 36, 1246-1252 (2007).

33. H. Watanabe et al., "Functional activation in diverse regions of the developing brain of human infants," NeuroImage 43, 346-357 (2008).

34. G. E. Strangman, Z. Li, and Q. Zhang, "Depth sensitivity and sourcedetector separations for near infrared spectroscopy based on the Colin27 brain template," PLoS One 8, e66319 (2013).

35. M. S. Beauchamp et al., "The developmental trajectory of brain-scalp distance from birth through childhood: implications for functional neuroimaging," PLoS One 6(9), e24981 (2011).

Biographies for the authors are not available. 